\title{
The Utility of Routine Chest Radiography in the Initial Evaluation of Adult Patients With Febrile Neutropenia Patients Undergoing HSCT
}

\author{
Deborah S. Yolin-Raley, PA-C ${ }^{a, *}$; Ibiayi Dagogo-Jack, MD b, ${ }^{b}$; Heidi B. Niell, PA-Ca; Robert J. Soiffer, MD; \\ Joseph H. Antin, MDª Edwin P. Alyea III, MDa ; and Brett E. Glotzbecker, MDa
}

Abstract

Background: The routine use of chest radiographs (CXRs) in the initial evaluation of asymptomatic patients with febrile neutropenia undergoing hematopoietic stem cell transplant (HSCT) is controversial. Objective: The goal of this study was to document the incidence of pneumonia demonstrated on CXR during an initial febrile neutropenic episode in adult patients undergoing HSCT. Materials and Methods: Clinical records of 1083 adult patients undergoing autologous $(n=766)$, allogeneic $(n=269)$, or umbilical cord blood HSCT ( $n=48)$ between October 1, 2009, and December 31, 2012, were retrospectively reviewed. CXRs obtained at the initial febrile neutropenic episode were evaluated for radiologic features of pneumonia. The presence of clinical symptoms, length of stay (LOS), and readmission rates were assessed. Results: A total of 817 (75\%) febrile neutropenic episodes were noted. Of the patients with neutropenic fevers, 455 (55\%) had CXRs. Of the 76 patients with respiratory symptoms at the time of CXR, 24 (31.6\%) had findings suggestive of pneumonia. None of the 379 CXRs performed in the absence of symptoms revealed an infectious process $(P=.0001)$. Moreover, the mean LOS was 23.8 days for patients receiving a CXR compared with 22.2 days $(P=.04)$ in patients without a CXR. Additionally, in patients who had CXRs, $15.7 \%$ were readmitted within 30 days compared with $7.4 \%$ in those without CXRs ( $P=.0004)$. Conclusions: Indiscriminate routine CXR at the time of first neutropenic fever in asymptomatic adults undergoing HSCT is unlikely to reveal an infectious process or change clinical practice, and may be associated with increased LOS and readmission rates. (J Natl Compr Canc Netw 2015;13:184-189)

\section{Background}

Most patients treated with myeloablative conditioning regimens before hematopoietic stem cell transplantation (HSCT) develop neutropenic fevers in the preengraftment period because of profound neutropenia. ${ }^{1,2}$ Fevers may signify potentially life-threatening

From the appartment of Medical Oncology, Dana-Farber/Brigham and Women's Cancer Center, and 'Department of Internal Medicine, Brigham and Women's Hospital, Boston, Massachusetts.

"These authors contributed equally to the study design and manuscript production.

Submitted January 30, 2014; accepted for publication May 28, 2014.

The authors have disclosed that they have no financial interests, arrangements, affiliations, or commercial interests with the manufacturers of any products discussed in this article or their competitors. This study was conducted as a routine quality initiative at the Dana-Farber Cancer Institute/Brigham and Women's Hospital. No grant support was used for this study or manuscript production. A portion of this work was presented at the American Society for Blood and Marrow Transplantation Tandem Meeting in February 2013.

Correspondence: Brett E. Glotzbecker, MD, Department of Medical Oncology, Dana-Farber/Brigham and Women's Cancer Center, 450 Brookline Avenue, Boston, MA 02115.

E-mail: brett_glotzbecker@dfci.harvard.edu infections. The degree and duration of neutropenia depends on the intensity of the conditioning regimen and the source of donor stem cells. ${ }^{3,4}$

Initial management of a febrile neutropenic episode involves obtaining at least 2 sets of blood cultures and immediate initiation of empirical broad-spectrum antibiotics with an antipseudomonal $\beta$-lactam agent., Current guidelines and evidence from retrospective trials in patients receiving chemotherapy or undergoing autologous and allogeneic HSCT suggest that additional investigational studies, particularly chest radiographs (CXRs), should be obtained in patients with clinical signs and symptoms suggestive of respiratory infection. ${ }^{7-11}$ However, the studies from which current guidelines were derived were primarily small, and involved pediatric populations. Because of the unique nature of adult transplant populations, the purpose of this quality improvement project was to investigate the incidence of pneumonia demonstrated on routine CXRs at the time of the initial febrile neutropenic episode in a group of patients undergoing autologous and allogeneic HSCT. 
Routine Chest Radiography During Neutropenia

\section{Materials and Methods}

We reviewed records of consecutive adult patients who underwent either myeloablative allogeneic, reduced-intensity/myeloablative umbilical cord blood transplantation (UCBT), or autologous HSCT at Dana-Farber/Brigham and Women's Cancer Center between October 1, 2009, and December 31, 2012. Patients undergoing reduced-intensity conditioning allogeneic HSCT were excluded from the analysis because they were typically discharged within 2 days of stem cell infusion. Fever was uniformly defined as an oral temperature greater than $100.4^{\circ} \mathrm{F}\left(38.0^{\circ} \mathrm{C}\right)$ or axillary temperature greater than $99.4^{\circ} \mathrm{F}\left(37.4^{\circ} \mathrm{C}\right)$. Neutropenia was defined as an absolute neutrophil count less than 500 cells/mcL. Symptoms of respiratory illness were documented, including cough, shortness of breath, and chest pain. Physical examination findings concerning for a pulmonary process included tachypnea (respiratory rate $>20$ breaths per minute) and hypoxia (oxygen saturation $<90 \%$ ); rales, rhonchi, and/or decreased breath sounds were also noted. At the onset of first neutropenic fever, blood cultures were drawn from the periphery and from all central lines. All patients received intravenous antibiotic coverage with cefepime, ceftazidime, piperacillin/tazobactam, or imipenem after blood cultures were drawn. Historically, CXRs were automatically ordered as part of the initial febrile neutropenia workup. As of July 2012, CXRs were ordered according to physician preference, instead of according to strict adherence to guidelines. Most of the CXRs ordered for the inpatients were obtained within 24 hours of initiation of antibiotics. Baseline characteristics and patient demographics were retrieved from the DanaFarber Cancer Institute (DFCI) HSCT repository. Data including the day of transplantation, patientreported symptoms, physical examination, and culture results were obtained from the electronic and paper medical records. CXRs were reviewed by staff radiologists at Brigham and Women's Hospital at the time of study completion. Abnormal CXRs were compared with the pre-HSCT films of the patients. Radiologic findings consistent with pneumonia, including the presence of confluent consolidative radiodensities, air bronchograms, and a lack of evidence of volume loss suggestive of atelectasis, were all included as possible cases of pneumonia. Additional thoracic imaging was obtained at the discretion of treating physicians. Descriptive statistics were used to compare baseline characteristics. Fisher exact test was used for 2 sample comparisons. Statistical significance was defined as a $P$ value less than .05. Analyses were performed using SAS software.

\section{Results}

\section{Patient Demographics}

A total of 1083 patients were admitted for HSCT between October 1, 2009, and December 31, 2012; 766 patients were admitted for autologous HSCT, 269 were admitted for myeloablative allogeneic HSCT, and 48 were admitted for either myeloablative or nonmyeloablative UCBT. Of the 766 patients admitted for autologous HSCT, 452 had multiple myeloma, 227 had non-Hodgkin's lymphoma (NHL), and 87 had Hodgkin lymphoma. This subgroup included 476 men and 290 women, and the median age was 61 years (range, $21-77$ years). Of the 269 patients admitted for allogeneic HSCT, $121 \mathrm{had}$ acute myeloid leukemia, 49 had acute lymphoblastic leukemia, and 44 had myelodysplastic syndromes/ myeloproliferative diseases; the remainder of the primary malignancies are discussed in Table 1 . This subgroup included 145 men and 124 women, and the median age was 46 years (range, $21-66$ years). Of the 48 patients admitted for double UBCT, most had acute myeloid leukemia $(n=19)$ or NHL $(n=13)$. This subgroup included 33 men and 15 women, and the median age was 53 years (range, 25-68 years). The primary malignancies of the patients admitted for HSCT are described in Table 1.

\section{Febrile Neutropenia}

A total of 825 febrile neutropenic episodes were noted in the 1083 patients (75.0\%). Of the 766 patients who underwent autologous HSCT, 547 (71.4\%) developed febrile neutropenia. Significantly more patients with lymphoma (276 of $314 ; 87.9 \%$ ) developed neutropenic fevers than those with myeloma (271 of $452 ; 60.0 \% ; P<.001$ ). The median day of first neutropenic fever was day +6 (range, day -9 to +12 ), with day 0 being the day of stem cell infusion. The number of patients with febrile neutropenia in the allogeneic HSCT group was 240 of 269 (89\%). The median day of first neutropenic fever was day +6 (range, day -8 to +13 ). In the 
Yolin-Raley et al

\begin{tabular}{|lccc|}
\hline Table 1 Primary Malignancies and Breakdown by Transplant Type \\
\hline Primary Malignancies & Autologous & Allogeneic $^{\text {a }}$ & Umbilical Cord Blood $^{\text {b }}$ \\
\hline ALL & - & 49 & 3 \\
AML & - & 121 & 19 \\
Aplastic anemia & - & 15 & 0 \\
CLL & - & 5 & 2 \\
CML & - & 12 & 2 \\
HLH & - & 1 & 0 \\
Non-Hodgkin's lymphoma & 227 & 18 & 13 \\
Hodgkin lymphoma & 87 & 1 & 1 \\
MDS/MPD & - & 44 & 8 \\
Multiple myeloma & 452 & 3 & 0 \\
Total & 766 & 269 & 48 \\
\hline
\end{tabular}

Abbreviations: ALL, acute lymphoblastic leukemia; AML, acute myeloid leukemia; CLL, chronic lymphocytic leukemia; CML, chronic myeloid leukemia; HLH, hemophagocytic lymphohistiocytosis; MDS, myelodysplastic syndromes; MPD, myeloproliferative diseases.

apatients who received myeloablative bone marrow or peripheral blood stem cell transplant.

${ }^{\text {b}}$ These patients underwent either myeloablative or reduced-intensity conditioning.

UCBT group, 38 of 48 patients (79\%) had febrile neutropenia, and the median day of first neutropenic fever was day +8 (range, day -7 to +16$)$.

\section{Chest Radiography}

Of the 825 patients evaluated with febrile neutropenia, 455 (55.2\%) had a CXR completed within 48 hours of their first temperature spike. Of the 547 patients undergoing autologous transplant who developed neutropenic fevers, 272 (49.7\%) had CXRs, 30 of which were performed in the setting of either respiratory symptoms or abnormal findings on examination. Of these 30 CXRs, 10 showed significant radiologic findings leading to new or continued management for a pulmonary infection. No significant difference was seen in the percentage of patients with respiratory symptoms or findings on physical examination who subsequently had evidence of pneumonia on CXR (40\% vs 20\%; $P=.43)$. Comparatively, none of the 242 CXRs $(P=.0001)$ performed in asymptomatic patients were concerning for a new infection.

More patients with febrile neutropenia received CXRs after allogeneic HSCT than after autologous HSCT (153 of 240; 63.8\%; $P=.002$ ). Only 41 patients who underwent allogeneic HSCT had documented respiratory findings, with 31 having physical findings suggestive of a pulmonary process and 10 having new symptoms, such as cough or shortness of breath. Of the 41 patients with findings, 12 (29\%) had new or worsening infiltrates on CXR. No significant difference was seen in the percentage of patients with respiratory symptoms or findings on examination who subsequently had evidence of pneumonia on CXR $(50 \%$ vs $33 \% ; P=.45)$. In contrast, none of the 112 CXRs $(P=.0001)$ performed in the absence of symptoms was concerning for infection. In the UCBT group, 30 of 38 patients (78.9\%) with febrile neutropenia underwent a CXR. This was significantly more than in the other 2 groups $(P=.002)$. Of the 30 patients, 5 who had CXRs had either a cough or hypoxemia; of these, 2 had multifocal pneumonia on CXR. None of the other 25 films $(P=.023)$ that were performed in the absence of symptoms revealed an infectious process. Table 2 summarizes the radiographic findings in the different groups.

\section{Patient Outcomes}

Length of stay (LOS) in the different populations was reviewed. In the patients who underwent myeloablative allogeneic or autologous HSCT, the shortest LOS was noted in the patients without an episode of febrile neutropenia, with a mean of 19.05 days. The mean LOS for patients who had fevers was 23.1 days $(P=.0001)$. When specifically considering the 425 patients who received a CXR at the time of the first temperature spike, the mean LOS was 23.8 days compared with 22.2 days $(P=.04)$ in the 362 patients who did not receive a $C X R$. In the patients who underwent 
Routine Chest Radiography During Neutropenia

Table 2 Evidence of PNA on CXR Obtained Within 48 Hours of Fever

\begin{tabular}{|c|c|c|c|c|}
\hline & $\begin{array}{l}\text { Total CXR } \\
\text { Ordered }\end{array}$ & $\begin{array}{l}\text { Reported PNA } \\
\text { With Respiratory } \\
\text { Symptoms or Signs }\end{array}$ & $\begin{array}{l}\text { Reported PNA With } \\
\text { No Respiratory } \\
\text { Symptoms or Signs }\end{array}$ & $P$ Value \\
\hline Autologous transplant & 272 & 10 of 30 & 0 of 242 & .0001 \\
\hline \multicolumn{5}{|l|}{ Allogeneic transplant } \\
\hline Myeloablative transplant ${ }^{a}$ & 153 & 12 of 41 & 0 of 112 & .0001 \\
\hline Umbilical cord blood transplant ${ }^{\mathrm{b}}$ & 30 & 2 of 5 & 0 of 25 & .0230 \\
\hline
\end{tabular}

Abbreviations: CXR, chest radiograph; PNA, pneumonia.

apatients who received peripheral blood or bone marrow transplants and did not receive umbilical cord blood transplants.

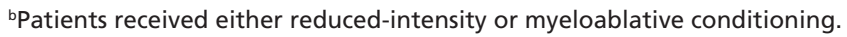

a UCBT, the shortest LOS of 30.7 days $(P=.04)$ was documented in those without fevers. Among patients with febrile episodes, the mean LOS was 45.8 days in those who had CXRs at the time of initial temperature spike. This was similar to the mean LOS of 44.6 days $(P=.89)$ noted in patients without documented CXRs.

Readmissions in the groups were also evaluated. In the patients who underwent myeloablative allogeneic and autologous HSCT, 19 of 248 patients (7.7\%) without fevers during neutropenia and 94 of 787 (11.9\%; $P=.06)$ with fevers were readmitted within 30 days. Among those with fevers who underwent a CXR, 67 of $425(15.7 \%)$ were readmitted, whereas only 27 of 362 (7.4\%; $P=.0004)$ without a CXR were readmitted within 30 days. Similarly, in the patients who received a UCBT, only 1 of $10(10 \%)$ without neutropenic fevers were readmitted within 30 days compared with 12 of 38 ( $31.5 \% ; P=.24)$ with fevers. In patients with fevers who received a CXR, 8 of $20(40 \%)$ were readmitted compared with 4 of 15 (26.6\%; $P=.49)$ without CXRs.

\section{Discussion}

Patients undergoing HSCT are at particularly increased risk for infections as a result of prolonged neutropenia and deficiencies in cellular and humoral immunity. At Dana-Farber/Brigham and Women's Hospital, all patients who develop febrile neutropenia while receiving a stem cell transplant undergo an extensive evaluation consistent with NCCN and Infectious Diseases Society of America guidelines, involving a detailed history and physical examination, blood and urine cultures, and site-specific cultures and/or imaging. 5.,612 Before October 31, 2011, the practice at our institution was to include a rou- tine CXR as part of the initial evaluation for patients with febrile neutropenia.

We conducted a retrospective investigation in a large cohort of adult patients that included both allogeneic and autologous HSCT recipients. Strikingly, none of the 379 CXRs obtained in asymptomatic individuals revealed radiographic findings of an infection. Moreover, all of the 24 patients with CXR findings concerning for infection had symptoms at the time that imaging was obtained. Importantly, most antibiotic regimens were not stopped or changed until the patient engrafted, regardless of CXR results.

The routine use of CXR in the initial evaluation of asymptomatic patients with febrile neutropenia is controversial. Minimal literature supports its utility, and the yield of conventional radiography is low in the early stages of pneumonia because of a diminished inflammatory response. ${ }^{10,13-15}$ The results presented herein were in concordance with those of most previous studies. Reports by several pediatric groups suggest that CXRs have limited benefit in children with routine febrile neutropenia after chemotherapy. ${ }^{8-10}$ In a study examining the utility of CXR during the initial febrile neutropenia episode in 81 pediatric patients receiving allogeneic HSCT, routine CXR only yielded evidence of a pulmonary infiltrate in $5(6 \%)$ of the patients. ${ }^{7}$ Similar results have been demonstrated in adult patients with febrile neutropenia in both the ambulatory ${ }^{16}$ and inpatient setting. ${ }^{17}$ Oude Nijhuis et $\mathrm{al}^{16}$ found that only 2 of 109 (1.8\%) CXRs were suggestive of pneumonia in ambulatory patients with febrile neutropenia without clinical signs of new pulmonary disease. Similarly, Navigante et $\mathrm{al}^{17}$ reported that only $2.3 \%$ of hospitalized oncology patients had CXR findings consistent with pneumonia in the absence of respiratory symptoms. 
Yolin-Raley et al

Contrary to the present findings, in a study of 61 adult patients who underwent autologous transplant, Roy et $\mathrm{a}^{18}$ reported that up to $31 \%$ of patients with abnormal CXRs had no clinical findings suggestive of pulmonary infection. The difference between the present results and those reported by Roy et $\mathrm{al}^{18}$ might be explained by the disparate patient populations and institution-specific antibiotic prophylactic protocols. At our institution, most of the patients who underwent autologous transplant had multiple myeloma, whereas most patients in the Roy et $\mathrm{al}^{18}$ study had breast cancer. All patients at our institution received prophylaxis with trimethoprimsulfamethizole, levofloxacin, acyclovir, and nystatin or clotrimazole troches, whereas prophylaxis in the study by Roy et $\mathrm{al}^{18}$ included ofloxacin, acyclovir, and fluconazole, but did not include trimethoprimsulfamethizole.

Looking specifically at the predictive values of respiratory symptoms, such as cough and shortness of breath, compared with respiratory signs on physical examination, such as rales and rhonchi, we found no significant difference in the percentage of patients with these findings who had evidence of pneumonia on CXR. Although the numbers are small, they suggest that CXRs should be considered in all patients with either a history of respiratory symptoms or abnormal physical examination findings.

It was also noted that both LOS and readmission rates in patients with fevers were significantly lower in those who did not undergo a CXR compared with those who did. A few factors could contribute to the differences in these important hospital metrics. The completion of radiologic studies depends on the availability of slots in radiology. A routine radiologic study ordered for fevers is usually completed and interpreted by a staff radiologist within 24 to 48 hours of order placement. Awaiting these results can lead to delays in care, such as changes or discontinuation of antibiotics. In addition, the development of new symptoms or signs without a cause elucidated on CXR requires further evaluation and possible intervention that may lead to further delays in discharge. With this, one may infer that the use of CXR during the initial febrile neutropenia episode in patients who underwent HSCT is a surrogate marker for acuity, which indirectly impacts LOS and readmission rates. This information could be used as part of a tool to predict discharge timing and risk of readmission.
The impact of LOS on outcome metrics such as cost has recently been evaluated in the HSCT population. In several studies, LOS has been shown to be a useful proxy for short-term costs. ${ }^{19,20}$ Each day a patient remains in the hospital impacts the overall cost, with baseline median costs ranging from $\$ 55,000$ to $\$ 75,000$ for an autologous HSCT and $\$ 150,000$ to $\$ 175,000$ for a myeloablative allogeneic HSCT. ${ }^{21}$ Furthermore, additional time in the hospital may increase the risk for hospital-associated complications, such as infection or venous thromboembolism.

Based on the initial observations that the utility of CXRs in diagnosing infection in the absence of symptoms was low and care outcomes were not improved as a result of the studies being ordered, we implemented an institution-wide policy limiting CXRs to symptomatic patients who underwent autologous HSCT. Using Lean Six Sigma and other process improvement techniques, the percentage of CXRs obtained at the initial febrile neutropenic episode in asymptomatic patients who underwent autologous transplant was reduced from 50\% to $5 \%$ during the first 6 months of the intervention. Before this intervention, the 1-year cost associated with ordering CXRs in asymptomatic patients with febrile neutropenia who underwent autologous HSCT was $\$ 43,420$. The savings associated with reducing the percentage of unnecessary CXRs to $5 \%$ in asymptomatic autologous patients with febrile neutropenia was approximately $\$ 38,858$. Applying the same rationale, in 2012, a total of 236 allogeneic HSCTs were performed at DFCI; reducing the number of CXRs in the allogeneic population would have assumed a cost savings of approximately $\$ 54,821$.

In addition to promoting institutional cost savings, the reduction in routine CXRs will lead to less radiation exposure to patients undergoing possibly curative therapy. In 1996, an estimated 52 CT scans were performed per 1000 people, and in 2010 that number increased to 149 per 1000 people..$^{22}$ Each CXR exposes a patient to $0.1 \mathrm{mSv}$ of radiation. This is equivalent to 10 days of exposure to natural causes of radiation, such as radon. ${ }^{23,24}$ Although the risk of subsequent malignancy is extremely low as a result of one CXR, each additional radiologic study increases the total lifetime risk. In addition to the risks of radiation exposure, unnecessary anxiety is provoked when studies report false-positive or 
equivocal findings that require further workup or intervention. Unless these radiologic studies can be shown to be associated with improved detection of infection or disease or to improve outcomes, careful thought should be given to ordering studies that may not be medically necessary.

\section{Conclusions}

Indiscriminate routine CXR at the time of first neutropenic fever in asymptomatic patients who have undergone HSCT is unlikely to reveal an infectious process or change clinical practice. Moreover, a reduction in the number of unnecessary CXRs ordered can lead to cost savings and reduced radiation exposure without compromising patient care.

\section{Acknowledgements}

The authors would like to thank all of the DFCI Bone Marrow Transplant Physician Assistants who helped make this quality initiative possible by actively managing the orders of their peers and physician moonlighters.

\section{References}

1. Browder AA, Huff JW, Petersdorf RG. The significance of fever in neoplastic disease. Ann Intern Med 1961;55:932-942.

2. Sickles EA, Greene WH, Wiernik PH. Clinical presentation of infection in granulocytopenic patients. Arch Intern Med 1975;135:715-719.

3. Sable CA, Donowitz GR. Infections in bone marrow transplant recipients. Clin Infect Dis 1994;18:273-281; quiz 282-284.

4. Copelan EA. Hematopoietic stem-cell transplantation. N Engl J Med 2006;354:1813-1826.

5. Baden LR, Bensinger W, Angarone M, et al. Prevention and treatment of cancer-related infections. J Natl Compr Canc Netw 2012;10:1412-1445.

6. Freifeld AG, Bow EJ, Sepkowitz KA, et al. Clinical practice guideline for the use of antimicrobial agents in neutropenic patients with cancer: 2010 update by the infectious diseases society of america. Clin Infect Dis 2011;52:e56-93.
7. Cox JA, DeMasi J, McCollom S, et al. The diagnostic utility of routine chest radiography in the evaluation of the initial fever in patients undergoing hematopoietic stem cell. Pediatr Blood Cancer 2011;57:666-668.

8. Renoult E, Buteau $\mathrm{C}$, Turgeon $\mathrm{N}$, et al. Is routine chest radiography necessary for the initial evaluation of fever in neutropenic children with cancer? Pediatr Blood Cancer 2004;43:224-228.

9. Roberts SD, Wells GM, Gandhi NM, et al. Diagnostic value of routine chest radiography in febrile, neutropenic children for early detection of pneumonia and mould infections. Supportive Care Cancer 2012;20:2589_ 2594.

10. Feusner J, Cohen R, O'Leary M, Beach B. Use of routine chest radiography in the evaluation of fever in neutropenic pediatric oncology patients. J Clin Oncol 1988;6:1699-1702.

11. Lehrnbecher $T$, Phillips $R$, Alexander $S$, et al. Guideline for the management of fever and neutropenia in children with cancer and/ or undergoing hematopoietic stem-cell transplantation. J Clin Oncol 2012;30:4427-4438.

12. Baden LR, Bensinger W, Angarone M, et al. NCCN Clinical Practice Guidelines in Oncology: Prevention and Treatment of Cancer-Related Infections. Version 2, 2014. Available at: NCCN.org. Accessed January $1,2014$.

13. Katz JA, Bash R, Rollins N, et al. The yield of routine chest radiography in children with cancer hospitalized for fever and neutropenia. Cancer 1991;68:940-943.

14. Korones DN, Hussong MR, Gullace MA. Routine chest radiography of children with cancer hospitalized for fever and neutropenia: is it really necessary? Cancer 1997;80:1160-1164.

15. Barloon TJ, Galvin JR, Mori M, et al. High-resolution ultrafast chest CT in the clinical management of febrile bone marrow transplant patients with normal or nonspecific chest roentgenograms. Chest 1991;99:928-933.

16. Oude Nijhuis CS, Gietema JA, Vellenga E, et al. Routine radiography does not have a role in the diagnostic evaluation of ambulatory adult febrile neutropenic cancer patients. Eur J Cancer 2003;39:2495-2498.

17. Navigante AH, Cerchietti LC, Costantini P, et al. Conventional chest radiography in the initial assessment of adult cancer patients with fever and neutropenia. Cancer Control 2002;9:346-351.

18. Roy V, Ali LI, Selby GB. Routine chest radiography for the evaluation of febrile neutropenic patients after autologous stem cell transplantation. Am J Hematol 2000;64:170-174.

19. Lee SJ, Zahrieh D, Alyea EP, et al. Comparison of T-cell-depleted and nonT-cell-depleted unrelated donor transplantation for hematologic diseases: clinical outcomes, quality of life, and costs. Blood 2002;100:2697-2702.

20. Lee SJ, Klar N, Weeks JC, Antin JH. Predicting costs of stem-cell transplantation. J Clin Oncol 2000;18:64-71.

21. Khera N, Zeliadt SB, Lee SJ. Economics of hematopoietic cell transplantation. Blood 2012;120:1545-1551.

22. Smith-Bindman R, Miglioretti DL, Johnson E, et al. Use of diagnostic imaging studies and associated radiation exposure for patients enrolled in large integrated health care systems, 1996-2010. JAMA 2012;307:24002409.

23. Little MP. Risks associated with ionizing radiation. $\mathrm{Br}$ Med Bull 2003;68:259-275.

24. Modan B, Chetrit A, Alfandary E, Katz L. Increased risk of breast cancer after low-dose irradiation. Lancet 1989;1:629-631. 